\title{
Refinement of the NHS locus on chromosome Xp22.13 and analysis of five candidate genes
}

\author{
Annick Toutain ${ }^{*}{ }^{1}$, Benoît Dessay ${ }^{1}$, Nathalie Ronce ${ }^{1}$, Maria-Immacolata Ferrante ${ }^{2}$, \\ Julie Tranchemontagne ${ }^{3}$, Ruth Newbury-Ecob ${ }^{4}$, Carina Wallgren-Pettersson ${ }^{5}$, \\ John Burn ${ }^{6}$, Josseline Kaplan ${ }^{7}$, Annick Rossi ${ }^{8}$, Silvia Russo ${ }^{9}$, Ian Walpole ${ }^{10}$, \\ James K Hartsfield ${ }^{11}$, Nina Oyen ${ }^{12}$, Andrea Nemeth ${ }^{13}$, Pierre Bitoun ${ }^{14}$, \\ Dorothy Trump $^{15}$, Claude Moraine ${ }^{1}$ and Brunella Franco ${ }^{2}$
}

${ }^{1}$ Service de Génétique, Hôpital Bretonneau, Centre Hospitalo-Universitaire, Tours, France; ${ }^{2}$ Telethon Institute of Genetics and Medicine, Naples, Italy; ${ }^{3}$ Medical Genetics Division, Children's Hospital, Montreal, Canada; ${ }^{4}$ Clinical Genetics Service, Institute of Child Health, Bristol Royal Hospital for Sick Children, Bristol, UK; ${ }^{5}$ Department of Medical Genetics, University of Helsinki, Finland; ${ }^{6}$ Institute of Human Genetics, Newcastle upon Tyne, UK; ${ }^{7}$ Service de Génétique Médicale, Hôpital Necker-Enfants Malades, Paris, France; ${ }^{8}$ EFS, Bois Guillaume, France; ${ }^{9}$ Laboratorio Biologia Molecolare, Instituto Auxologico Italiano, Milano, Italy; ${ }^{10}$ Genetic Services, Princess Margaret Hospital for Sick Children, Perth, Australia; ${ }^{11}$ Department of Oral Facial Development, Oral Facial Genetics Section, Indiana University School of Dentistry, Indianapolis, Indiana, USA; ${ }^{12}$ Center for Medical Genetics and Molecular Medicine, Haukeland University Hospital, Bergen, Norway; ${ }^{13}$ Oxford Clinical Genetic Service, Department of Clinical Genetics, Oxford Radcliffe Hospital NHS Trust, Oxford, UK; ${ }^{14}$ SIDVA 91, Savigny sur Orge, France; ${ }^{15}$ Department of Medical Genetics, Cambridge Institute for Medical Research, University of Cambridge, UK

Nance-Horan syndrome (NHS) is an X-linked condition characterised by congenital cataracts, dental abnormalities, dysmorphic features, and mental retardation in some cases. Previous studies have mapped the disease gene to a $2 \mathrm{cM}$ interval on Xp22.2 between DXS43 and DXS999. We report additional linkage data resulting from the analysis of eleven independent NHS families. A maximum lod score of $9.94(\theta=0.00)$ was obtained at the RS1 locus and a recombination with locus DXS1195 on the telomeric side was observed in two families, thus refining the location of the gene to an interval of around $1 \mathrm{Mb}$ on Xp22.13. Direct sequencing or SSCP analysis of the coding exons of five genes (SCML1, SCML2, STK9, RS1 and PPEF1), considered as candidate genes on the basis of their location in the critical interval, failed to detect any mutation in 12 unrelated NHS patients, thus making it highly unlikely that these genes are implicated in NHS.

European Journal of Human Genetics (2002) 10, 516-520. doi:10.1038/sj.ejhg.5200846

Keywords: Nance-Horan syndrome; chromosome Xp22.13; genetic mapping; candidate gene analysis

\section{Introduction}

Nance-Horan syndrome (NHS) is a rare X-linked disorder ${ }^{1-3}$ characterised by (1) bilateral congenital cataracts associated with microcornea and usually responsible for severe visual impairment; (2) distinctive dental anomalies, mainly

\footnotetext{
*Correspondence: A Toutain, Service de Génétique, Hôpital Bretonneau, 2 boulevard Tonnellé, 37044 Tours cedex 1, France. Tel: + 332474747 99; Fax: +33 2474786 53; E-mail: toutain@med.univ-tours.fr

Received 19 February 2002; revised 7 May 2002; accepted 13 May 2002
}

unusual dental morphology; (3) characteristic facial features; and (4) mental impairment in $30 \%$ of cases. ${ }^{4}$ Heterozygous females show milder signs of the disease, typically posterior Y-sutural lens opacities and/or slight dental anomalies. Linkage studies have localised the NHS gene to $\mathrm{Xp} 22.2^{5,6}$ and more recent unpublished data have placed the gene in a $2 \mathrm{cM}$ interval between markers DXS43 and DXS999 (Javed et al, personal communication). Francis et al. ${ }^{7}$ recently identified a locus for isolated Xlinked cataract (without the other features of NHS but with 


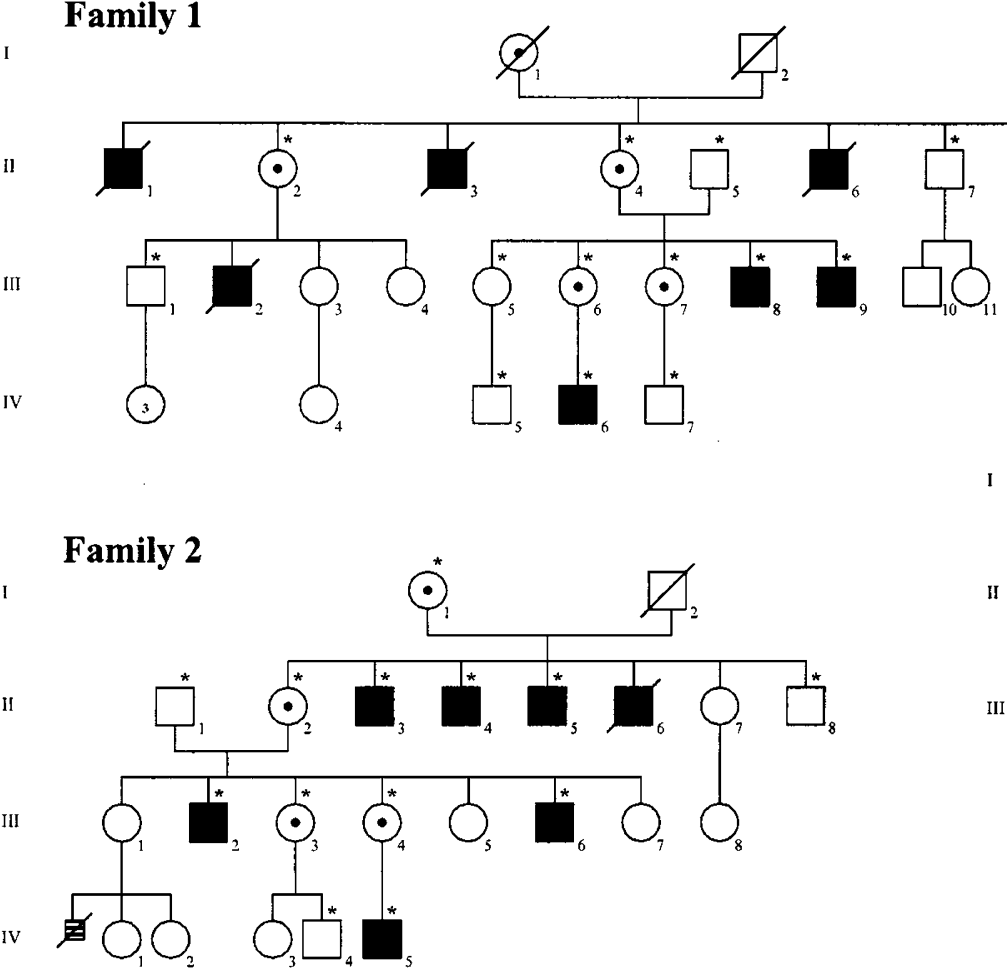

Family 4
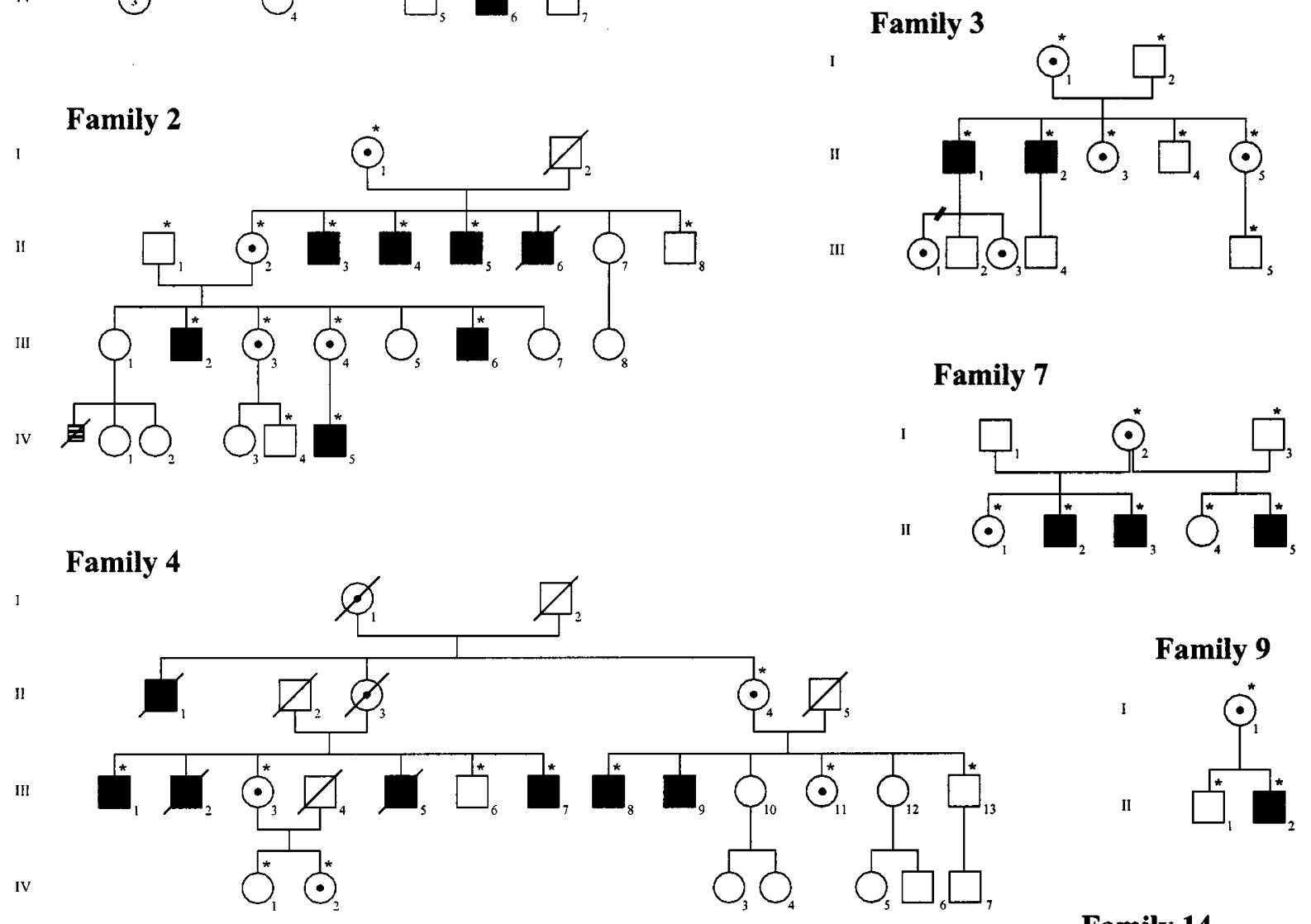

Family 14

Family 8

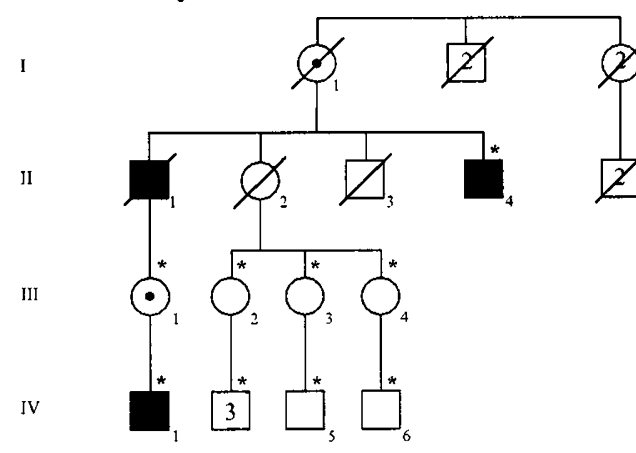

Family 10

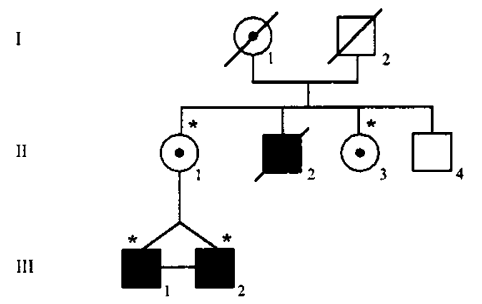

Family 9

1

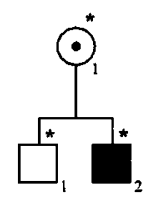

Figure 1 Pedigrees of the eleven families included in the linkage analysis. Filled squares denote affected males and dotted circles denote heterozygous females. Female carrier status was assigned from family history (obligate carriers) or from clinical signs (lens opacities and/or typical dental anomalies, in most cases limited to infraclinical findings). 
Table 1 Results of pairwise Lod scores

\begin{tabular}{llllllll}
\hline \multirow{2}{*}{ Locus } & 0.00 & 0.01 & 0.05 & 0.1 & 0.2 & 0.3 & 0.4 \\
\hline DXS43 & 6.04 & 5.91 & 5.45 & 4.85 & 3.62 & 2.32 & 1.02 \\
DXS1195 & $-\infty$ & 3.46 & 4.33 & 4.25 & 3.43 & 2.27 & 1.01 \\
DXS418 & 9.84 & 9.65 & 8.87 & 7.86 & 5.74 & 3.53 & 1.41 \\
RS1 & 9.94 & 9.75 & 8.96 & 7.93 & 5.75 & 3.42 & 1.17 \\
DXS999 & 7.25 & 7.10 & 6.52 & 5.79 & 4.28 & 2.71 & 1.19 \\
DXS7163 & 9.60 & 9.41 & 8.62 & 7.60 & 5.45 & 3.19 & 1.13 \\
\hline
\end{tabular}

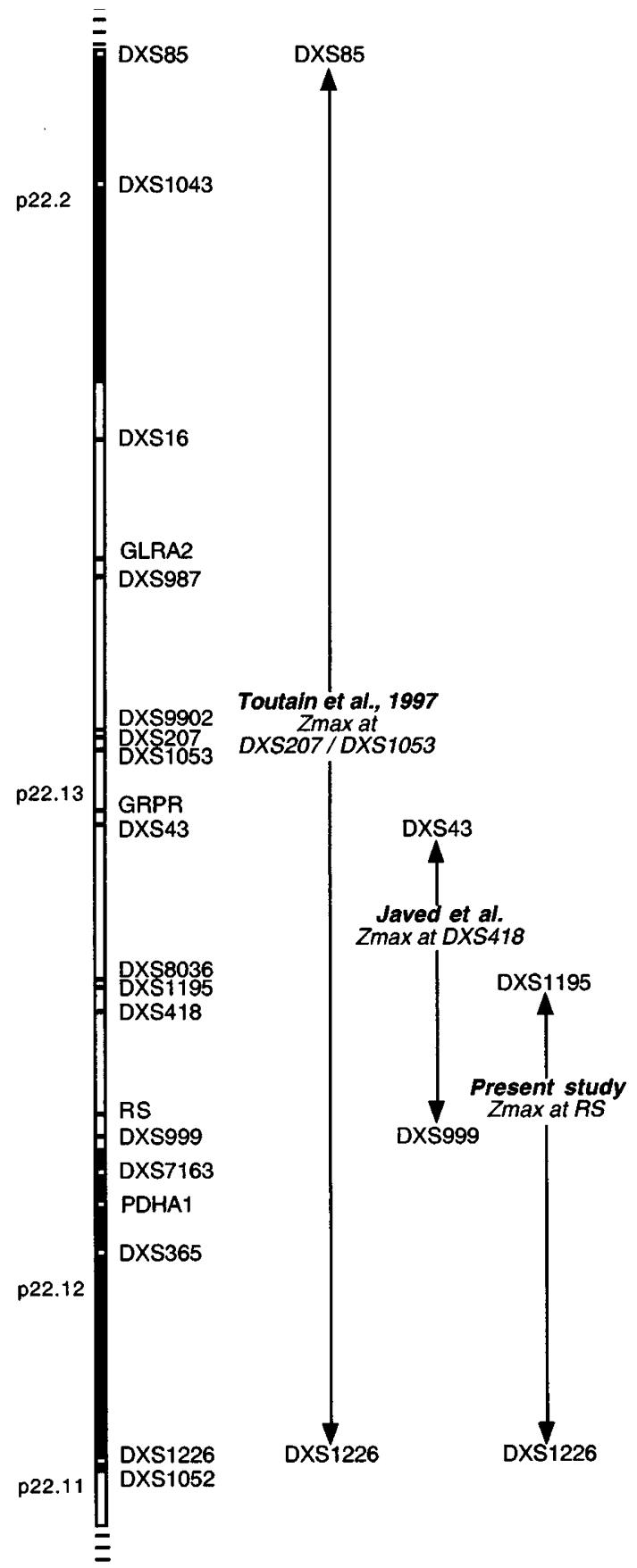

cardiac abnormalities in some patients) mapping to $\mathrm{Xp} 22$ (Zmax for DXS8036), within a $3 \mathrm{cM}$ interval flanked by DXS9902 and DXS999 and encompassing the NHS interval. This suggests allelic heterogeneity and strongly supports the existence of a gene for congenital cataract in this Xp region. Several genes have been identified in this region and we previously reported the exclusion of RAI2 as the causative gene for NHS. ${ }^{6}$ Here we report the results of linkage studies performed in 11 multiplex families refining the localisation

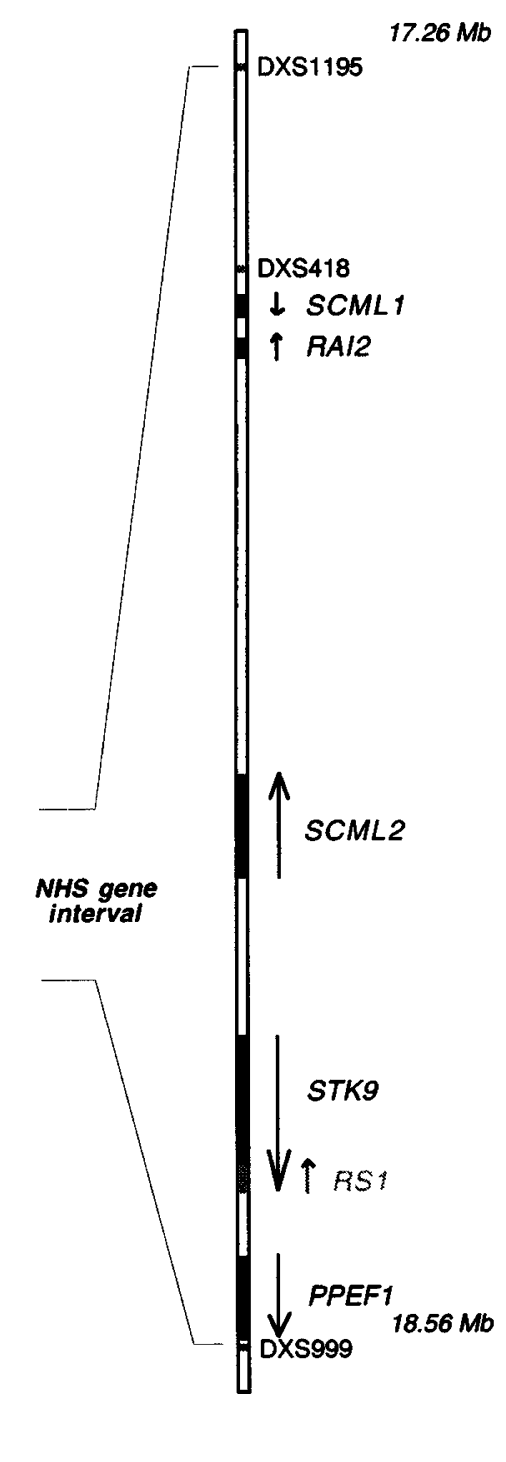

Figure 2 Schematic map of the Xp22.13 region, linkage intervals and schematic position of the candidate genes. 
of the gene on the telomeric side. We also report the results of sequencing and SSCP analysis of five genes, SCML1, SCML2, STK9, RS1 and PPEF1, considered as candidates on the basis of their position in the critical interval.

\section{Patients, materials and methods}

Thirteen independent multiplex NHS families and three sporadic cases (two males and one symptomatic heterozygous female) were recruited from various countries in the world, including previously reported cases. ${ }^{3,8}$ Male patients and heterozygous females were diagnosed according to precise clinical criteria as previously reported. ${ }^{5}$

DNA was extracted from blood lymphocytes of each consenting family member according to standard procedures. Microsatellite marker assay and linkage analysis were performed as previously described. ${ }^{5}$ Linkage analysis was performed in 11 multiplex families, including the four families tested in an earlier study ${ }^{5}$ in which additional markers and additional individuals (III-5 in family 3 and III-11 in family 4) were analysed (Figure 1).

Mutation screening in all coding exons of the five candidate genes was performed in 12 unrelated NHS patients. DNA was amplified by polymerase chain reaction (PCR) by using $250 \mathrm{ng}$ template DNA, 50 pmoles gene-specific primers (primer sequences are available upon request) and 2.5 U Taq DNA polymerase (Promega). The PCR products were analysed by SSCP analysis (GenePhor system, Amersham-Pharmacia) for STK9 (20 exons) and by sequencing using a PRISM BigDye Terminator Cycle sequencing kit and an ABI 377 automated DNA sequencer (Perkin-Elmer Applied Biosystems) for SCML1 (6 exons), SCML2 (15 exons), RS1 (6 exons) and PPEF1 (15 exons).

\section{Results and discussion}

Linkage analysis showed that the disease-causing gene mapped to the Xp22 region in all families tested and a combined maximum two-point lod score of 9.94 at $\theta=0$ was obtained at the RS1 locus (Table 1). A recombination with the marker DXS1195 was observed in two independent families, refining the localisation of the NHS gene on the telomeric side. These results combined with the previous data reduce the NHS gene interval to around $1 \mathrm{Mb}$ between DXS1195 on the telomeric side and DXS999 on the centromeric side (Figure 2). In view of the recent knowledge on the human genome sequence, ${ }^{9}$ the NHS gene is now placed in the Xp22.13 region.

Six genes (SCML1, RAI2, SCML2, STK9, RS1 and PPEF1) have already been identified in this newly defined interval and were considered as candidates for NHS by virtue of their position (Figure 2). Although RS1 is expressed exclusively in the sensory neuroretina and is responsible for $\mathrm{X}$ linked juvenile retinoschisis, ${ }^{10}$ an ocular condition different from the eye problems observed in NHS, it was considered as a possible candidate because the maximum lod score was obtained at this locus. None of the other five genes located in the NHS critical interval has so far been associated with a human condition. We previously reported that no mutations were found in RAI2 (retinoic-acidinduced 2) in nine NHS patients. ${ }^{6}$ SCML1 and SCML2 (sex comb on midleg-like-1 and 2), which are mammalian homologues to Scm, a member of the Drosophila Polycomb group of genes, ${ }^{11,12}$ are probably involved in transcriptional repression of HOX genes, and mutations in these genes may cause developmental malformations (though not similar to the spectrum of anomalies seen in NHS). However, their precise role in human embryo development remains to be elucidated. STK9 (serine-threonine kinase 9) encodes a serine-threonine protein kinase expressed in a variety of tissues, ${ }^{13}$ and there are several examples, such as CoffinLowry syndrome, showing that serine-threonine kinases may be involved in the pathogenesis of genetic disorders. The PPEF1 (protein phosphatase with E-F hand motif) gene is highly homologous to the Drosophila retinal degeneration $\mathrm{C}$ (rdgC) gene and encodes a serine-threonine phosphatase which is selectively expressed in sensory neurons of the dorsal root ganglia and neural crest-derived cranial ganglia. ${ }^{14}$ The coding sequence of SCML1, SCML2, STK9, RS1 and PPEF1 was analysed in 12 unrelated NHS patients. No mutations or polymorphic sequence changes were identified in any of the patients tested. On the basis of these results we conclude that it is very unlikely that any of these five genes is involved in NHS and further analysis will be necessary to identify the molecular basis of this condition. Similarly, SCML1, SCML2, PPEF1 and STK9 remain candidate genes for other diseases mapping to Xp22.13.

\section{References}

1 Nance WE, Warburg M, Bixler D, Helveston EM: Congenital Xlinked cataract, dental anomalies, and brachymetacarpalia. Birth Defects OAS 1974; 10: $285-291$.

2 Horan MB, Billson FA: X-linked cataract and Hutchinsonian teeth. Aust Paediat J 1974; 10: $98-102$.

3 Walpole IR, Hockey A, Nicoll A: The Nance-Horan syndrome. $J$ Med Genet 1990; 27: 632-634.

4 Toutain A, Ayrault AD, Moraine C: Mental retardation in NanceHoran syndrome: clinical and neuropsychological assessment in four families. Am J Med Genet 1997; 71: 305-314.

5 Toutain A, Ronce N, Dessay B et al: Nance-Horan syndrome: Linkage analysis in four families refines localization in Xp22.31p22.13 region. Hum Genet 1997; 99: 256-261.

6 Walpole SM, Ronce N, Grayson C et al: Exclusion of RAI2 as the causative gene for Nance-Horan syndrome. Hum Genet 1999; 104: $410-411$

7 Francis PJ, Berry V, Hardcastle AJ, Maher ER, Moore AT, Bhattacharya SS: A locus for isolated cataract on human Xp. J Med Genet 2002; 39: 105-109.

8 Bixler D, Higgins M, Hartsfield J: The Nance-Horan syndrome: a rare X-linked ocular-dental trait with expression in heterozygous females. Clin Genet 1984; 26: 30-35.

9 Ensembl Genome Server: http://www.ensembl.org.

10 Sauer CG, Gehrig A, Warneke-Wittstock R et al: Positional cloning of the gene associated with X-linked juvenile retinoschisis. Nat Genet 1997; 17: $164-170$. 
11 van de Vosse E, Walpole SM, Nicolaou A et al: Characterization of SCML1, a new gene in Xp22, with homology to developmental polycomb genes. Genomics 1998; 49: 96-102.

12 Montini E, Buchner G, Spalluto C et al: Identification of SCML2, a second human gene homologous to the Drosophila sex comb on midleg $(\mathrm{Scm})$ : a new gene cluster on Xp22. Genomics 1999; 58: $65-72$.
13 Montini E, Andolfi G, Caruso A et al: Identification and characterization of a novel serine-threonine kinase gene from the Xp22 region. Genomics 1998; 51: 427-433.

14 Montini E, Rugarli EI, van de Vosse E et al: A novel human serinethreonine phosphatase related to the Drosophila retinal degeneration $\mathrm{C}(\mathrm{rdgC})$ gene is selectively expressed in sensory neurons of neural crest origin. Hum Mol Genet 1997; 6: 1137-1145. 